\section{OPEN ACCESS}

Approved by:

Frontiers in Chemistry Editorial Office,

Frontiers Media SA, Switzerland

*Correspondence:

Luyun Cai

lycai515@163.com

Yanbo Wang

wangyb@mail.zjgsu.edu.cn

tThese authors have contributed equally to this work

¥Co-first author

Specialty section:

This article was submitted to

Food Chemistry,

a section of the journal

Frontiers in Chemistry

Received: 31 May 2018

Accepted: 15 February 2019

Published: 02 April 2019

Citation:

Nian L, Cao A, Wang J, Tian H, Liu Y,

Gong L, Cai L and Wang Y (2019)

Corrigendum: Viscoelastic and

Functional Properties of Cod-Bone

Gelatin in the Presence of Xylitol and

Stevioside. Front. Chem. 7:120

doi: 10.3389/fchem.2019.00120

\title{
Corrigendum: Viscoelastic and Functional Properties of Cod-Bone Gelatin in the Presence of Xylitol and Stevioside
}

\section{Linyu Nian ${ }^{1,2 t}$, Ailing Cao ${ }^{2,3 t \neq}$, Jing Wang ${ }^{2}$, Hongyu Tian², Yongguo Liu' ${ }^{2}$, Lingxiao Gong ${ }^{2}$, Luyun Cai ${ }^{1,2 *}$ and Yanbo Wang ${ }^{4 *}$}

${ }^{1}$ College of Food Science and Engineering, Bohai University, National \& Local Joint Engineering Research Center of Storage, Processing and Safety Control Technology for Fresh Agricultural and Aquatic Products, Jinzhou, China, ${ }^{2}$ Beijing Advanced Innovation Center for Food Nutrition and Human Health, Beijing Technology and Business University, Beijing, China, ${ }^{3}$ Hangzhou Customs District, Hangzhou, China, ${ }^{4}$ College of Food Science and Biotechnology, Zhejiang Gongshang University, Hangzhou, China

Keywords: bone gelatin, xylitol, stevioside, Gadus morhua, cod

\section{A Corrigendum on}

Viscoelastic and Functional Properties of Cod-Bone Gelatin in the Presence of Xylitol and Stevioside

by Nian, L., Cao, A., Wang, J., Tian, H., Liu, Y., Gong, L., et al. (2018). Front. Chem. 6:111. doi: $10.3389 /$ fchem.2018.00111

In the published article, an author name was incorrectly spelled as "Yuhao Wang." The correct spelling is "Yanbo Wang."

Additionally, there was an error in affiliation 2. Instead of "Xiaoshan Entry-Exit Inspection and Quarantine Bureau, Hangzhou, China," it should be "Hangzhou Customs District, Hangzhou, China."

Lastly, there was also an error regarding the affiliations for Linyu Nian and Luyun Cai. They should also have the affiliation "Beijing Advanced Innovation Center for Food Nutrition and Human Health, Beijing Technology and Business University, Beijing, China” and Yanbo Wang should not have affiliation 3 .

The authors apologize for this error and state that this does not change the scientific conclusions of the article in any way. The original article has been updated.

Copyright $\odot 2019$ Nian, Cao, Wang, Tian, Liu, Gong, Cai and Wang. This is an open-access article distributed under the terms of the Creative Commons Attribution License (CC BY). The use, distribution or reproduction in other forums is permitted, provided the original author(s) and the copyright owner(s) are credited and that the original publication in this journal is cited, in accordance with accepted academic practice. No use, distribution or reproduction is permitted which does not comply with these terms. 